\title{
Mechanisms and Patterns of Grammatical Replication Caused by Kanbun Kundoku
}

\author{
Hongyan Liu ${ }^{1, a^{*}}$, Yujie Zhao ${ }^{2, b}$, \\ ${ }^{1}$ School of Foreign Languages, Yanshan University, China \\ ${ }^{2}$ School of Foreign Languages, Central China Normal University, China \\ aliuhongyan@ysu.edu.cn, b1147908019@qq.com
}

\begin{abstract}
Keywords: grammatical replication, language contact, Kanbun Kundoku.
\end{abstract}
\begin{abstract}
This paper is about the grammatical evolution caused by language contact and its mechanisms. From the discussion, we concluded that the grammatical replication is an interactive effect both from outside influence and inside changes. The grammatical evolution in East Asian languages caused by Kanbun Kundoku could be seen as an outside inducer. These grammatical function and meaning from Chinese language can be in localization to cause changes in grammatical form of other East Asian languages. Grammatical replication's main motivation is the formulas of equivalence in languages. Based on this rule, after the grammatical rules in Chinese putting into the other languages, it can localize East Asian languages' grammatical system and typology to make it as compatible grammatical component.
\end{abstract}

\section{Introduction}

Kanbun Kundoku is a kind of language contact phenomenon which is common in East Asia. Kunten marks were often made during the process of vernacular reading of Chinese Confucianist literature and Buddhist texts in ancient Japan, Korean Peninsula and Vietnam etc. Chinese had an impact on ancient local languages. As a result, these influences have gradually become an important part of these languages.

There are two main explanations of East Asian languages evolution mechanism caused by Kanbun Kundoku. One is "grammaticalization", the other "grammar Borrowing". On the one hand, Naruse-Shima (1997) and Matsumoto (1998) illustrated in their Japanese Postpositions studies that evolution path can be seen as a type of continuum of clines although Postpositions are generated in Kunten literatures, so it can be understood that these series of grammatical markings are generated by inner language mechanisms of analogy or reanalysis. In spite of influence of Chinese grammar, it is a grammaticalization process of grammar markings' generative process like Japanese postpositions. On the other hand, it is believed by Zisk (2013) that other East Asian languages have absorbed part of Chinese grammar during Kanbun Kundoku process. This change can be seen as a sort of loan translation, and loan pos-shift or loan syntax from the perspective of mechanism, not referring to inner changes in target languages.

Inner changes and exterior influences in languages are opposite from these two views primarily. However, from recent linguistic research studies, there is the chance of compatibility of inner changes and exterior influences. From the history of East Asian languages evolution caused by Kanbun Kundoku, those languages have had inner changes based on exterior impacts from the outside. The example of Kanbun Kundoku influencing East Asian languages is taken by this paper to explain the following questions. The first question is that whether there be a clear boundary between inner changes and exterior influences of languages. If it did not exist, what kind of relationship of these two could be. The second question is that if language evolution caused by language contacts was a common phenomenon or a systematic mechanism. The third question is that it is unsure that language contacts can accelerate language inner evolution or an important inducement of one language evolution itself. This paper will focus on these questions. 


\section{Grammar Evolution Caused by both Interior and Exterior Influences}

It is believed by general linguistic perspectives that language has a steady inner system which is determined by typology features of language itself and it will not have fundamental changes affected by the outside. Meillet (1977:76) and Bynon (1977:239) have pointed out that the form of one language affected by another language is that it will have lexical changes by lexical Borrowing but syntactic grammar markings and syntactic structure will not change restricted by phonology and morphology. Nonetheless, from the facts of Kanbun Kundoku, Chinese does not only influence East Asian words but also syntactic aspects.

Language contact researches in recent years have more reasonable explanations for this question. The researches which were conducted by Thomason (2001:71) and Winford (2007:23) illustrate that, except for language evolution caused by inner mechanism, there is language contact induced change phenomenon. From this point of view, language evolution induced by language contact can be divided into following hierarchies called as "Borrowing hierarchy" or "Borrowing scale"

\section{Lexicon content words > Lexicon Function words / Grammatical marking > Phonemes structure / Grammar structure $>$ Morpheme category}

Those language evolutions happen in hierarchy influenced by language contact. Lexical part is the easiest one to change during the influences of other languages in comparison with grammar, phonology and morphology which is harder to have a change. The different Borrowing hierarchy is decided by length and intensity of language contact. Kanbun Kundoku is high-intensive language contact for a long time. East Asian languages are influenced by ancient Chinese in many aspects during the process of Kanbun Kundoku. Not only did many Chinese Borrowing words occur, but also the system of phonology, grammar and morphology has changed. It is sure that the process of Kanbun Kundoku is in a high hierarchy of Borrowing scale. This is different from normal Borrowing because it is not simple additions of an inventory in target languages but a localization process which happen when Borrowing components put into target languages. This change is triggered by language contact, seen as vernacular motivation.

From the examples of Kanbun Kundoku, the localization process can be divided into these categories. In the first place, Borrowing Chinese element can become a new element when target languages' suffixes are added. For example, content words in Chinese cannot be used directly in target languages, like -zu, -su, -fu and - $k u$ etc. As a result, those are put into Japanese agglutinative grammar system. In the second place, some certain language evolution paradigm are sent to target languages as a whole, generating new grammar system, for example, there is a function conversion relationship between ancient Chinese adjectives and passive verbs like Di, Xiao, Chang, Qing and An. What's more, the paradigm of Adj $>V t$ happens in Japanese influenced by this. This relationship of normal form in Borrowing use agglutinative form like -kusu, -misu to realize Borrowing components' Borrowing. In the third place, general grammar rules cannot meet needs with a large amount of Chinese words Borrowing. One grammar rule have been normal that the borrowed verb can be further developed by adding suffix, -su during process of analogy of Japanese.

From the analysis above, it can be concluded that target languages' grammar evolution caused by Kanbun Kundoku is a result of interactive changes both from exterior influences and language inner changes. The outside influence is an inducer of language evolution. When grammar elements in Chinese are borrowed by target languages, there must be a process of localization to make it fit target languages' typology in morphology and syntactic aspects and become a part of target languages.

\section{Mechanism and Motivation of Grammar Duplication}

Grammar evolution caused by language contact can be divided into two types: grammar marking Borrowing and grammar replication. Grammar marking Borrowing refers to the direct input and use of other languages' grammar markings. It can be seen as the derivation of lexical Borrowing which is function words Borrowing in essence. Nonetheless, the input object of grammatical replication is not 
grammatical element in language, but another language's grammatical model. Heine\&Kuteva (2005:2) pointed out status of grammatical replication in grammatical Borrowing. The objects of grammatical Borrowing can be divided as follows:

Kinds of linguistic transfer

a. Form, that is, sounds or combinations of sounds

b. Meanings (including grammatical meanings or functions) or combinations of meanings

c. Form - meaning units or combinations of form - meaning units

d. Syntactic relations

In those Borrowing forms, (a) and (c) can be seen as function word's Borrowing category. However, Borrowing contents in (b) and (d) do not belong to grammar. They are a sort of model or paradigm. Therefore, the grammatical replication is a kind of grammatical model or paradigm when one language has an influence on objective language. What's more, a new grammatical function or syntactic structure can be formed by using fixed element in one language.

Kanbun Kundoku influences on East Asian languages are mainly in Borrowing, but this grammatical phenomenon is widespread. What's more, the same grammar form in Chinese even can be absorbed by both Borrowing and grammatical replication in other languages. For instance, naizhi in ancient Chinese can be borrowed as normal adverb naishi through Kunten literature into Japanese, and its grammatical function and meaning have been in reservation. At the same time, grammatical replication also occurred in naizhi's Japanese Borrowing. It duplicates by fixed agglutinative form in Japanese and only absorbs its grammatical function and meaning. Through Japanese localization, it has become the auxiliary word of itarumade and use fixed Japanese adverb, sunawachi, to emphasize this word. During the process of localization, the basic meaning and function of naizhi are the same but the concrete form in objective language has changed. Therefore, naishi and sunawachi itarumade are all borrowed from the same form of Chinese grammar. The former is borrowed from grammatical marking, the latter is a replication of one grammatical model. Another example is that suo in Chinese is a common nominalizing suffix. The localization of that nominalization grammatical function is realized by Kanbun Kundoku based on Borrowing suo- $V$ in Japanese content words. In this process, it not only generates the Borrowing form of sho- $V$, but also use nominalizing suffix in Japanese itself to duplicate grammatical function as agglutinative form of $V$-tokoro, even further developing as a new complementizer.

From those examples of Kanbun Kundoku, grammatical replication is a common phenomenon. Target languages duplicate grammar items of model or paradigm in other languages through language contact to make grammar function and grammatical meanings be in localization. Grammatical replication category is very widespread during this process. The objects of replication include single grammatical marking, one type of grammar, word formation rules, the syntactic function of function word and syntactic structure.

\section{Motivation and Mechanism in Grammatical Replication}

Grammatical replication is a process of localization in model or paradigm which has been borrowed in grammatical meanings and functions in target languages. Its mechanism is complicated because it

varies by differences in typology features of different languages. First of all, it is a worthwhile question to think about motivations in target languages' localization materials and grammatical forms of source languages.

Keesing (1991:316) pointed out that the grammatical conversion of two languages with different typology features is based on a kind of formulas of equivalence when they make language contact. Based on this rule, grammatical from in target languages can make evolution and identification with source languages' grammatical form. To conclude all studies, the concept of formulas of equivalence can be defined as the $\mathrm{X}$ language element in objective language has similar points with $\mathrm{Y}$ language 
element in source language. The $\mathrm{X}$ can be explained by $\mathrm{Y}$ based on the same cognitive structure in language contact to realize the localization of its function and meaning.

Ideographical expression feature in Chinese has an important impact on the realization of formulas of equivalence in Kanbun Kundoku. The equivalence between $\mathrm{X}$ in Chinese and $\mathrm{Y}$ in another language is established by East Asian people's understanding about Chinese characters. The grammatical equivalence between languages in grammatical replication is not the form, but semantics and functions etc. This also induces cognitive mechanism in grammatical replication.

The equivalence in semantics is always based on content word in Kanbun Kundoku. For instance, Chinese preposition of yin has been in localization as postpostion marking Japanese yotte which has the same function and meaning. During this process, the equivalence between yotte and yin are based on verb in semantics. Besides, they all have the meaning of "close to, rally on". Yotte is selected as corresponding form by semantic similarities. Therefore, the postposition word yotte which shows the reason has been generated in Japanese. Besides, the grammatical equivalence in grammatical function is a reconstitution process about function category of Borrowing grammatical items in target languages. The function category may be changed when a grammatical form is replicated because the grammatical features in typology of source language and objective language are different, for example, when the preposition yin and yi replicate into Japanese, there is no preposition words in Japanese typology feature, so it is realized by junctions and postpostions through adding agglutinative suffixes such as yotte and motte. Then the equivalence in grammatical marking has been realized.

From the above analysis, the main motivation in grammatical replication is formulas of equivalence existing in languages. By this formula of equivalence, when grammatical from in source language puts into target language, it will localize into compatible grammatical meaning and functional categories with target languages' grammatical system and typology. Based on this, grammatical replication mechanism can be summarized referred to Heine\&Kuteva $(2005: 81)$ as follows:

a. Speakers notice that in language $M$ there is a grammatical category $\mathrm{Mx}$.

b. They create an equivalent category $\mathrm{Rx}$ in language $\mathrm{R}$ on the basis of the use patterns available in $\mathrm{R}$.

c. To this end, they draw on universal strategies of formulas of equivalence, using construction Ry in order to develop Rx.

d. They replicate $\mathrm{Ry}$ to $\mathrm{Rx}$

\section{Summary}

It can be known from this paper's discussion that grammatical evolution caused by language contact is an interactive effect both from outside influence and inside changes. In the process of grammatical evolution in East Asian languages which is caused by Kanbun Kundoku, the influence of Chinese can be seen as an outside inducer. The process of localization happens when Chinese grammatical elements are borrowed by target languages to make it fit target languages' typology features in syntactic and morphology aspects, making it as a part of objective language.

Grammatical replication is a widespread phenomenon. The difference between grammatical Borrowing is that the object of grammatical replication is grammatical items like model or paradigm in other languages. These grammatical function and meaning can be in localization to cause changes in grammatical form.

Grammatical replication's main motivation is the formulas of equivalence in languages. Based on this rule, after the grammatical rules in source language putting into target languages, it can localize target languages' grammatical system and typology to make it as compatible grammatical component.

\section{Acknowledgement}

This work was supported by MOE (Ministry of Education in China) Project of Humanities and Social 
Sciences (No.16YJC740044), the Humanities and Social Sciences Projects of Hebei Provincial Department of Education (No.SD161100), and Young Teacher Research Project of Yanshan University (No.15SKB003).

\section{References}

[1] T. Bynon, Historical linguistics: Cambridge University Press. 1977.

[2] B. Heine, T. Kuteva, Language Contact and Grammatical Change: Cambridge University Press, 2005.

[3] R. M. Keesing, Substrates, Calquing and Grammaticalization in Melanesian Pidgin. Approaches to Grammaticalization: John Benjamins.pp 315-342.1991.

[4] Y. Matsumoto, "Semantic Change in the Grammaticalization of Verbs into Postpositions in Japanese." Studies in Japanese Grammaticalization : Cognitive and Discourse Perspectives: Kurosio, pp25-60, 1998.

[5] S. G. Thomason, Language Contact: Edinburgh University Press. 2001.

[6] S. G. Thomason, T. Kaufman, Language Contact, Creolization, and Genetic Linguistics: University of California Press, 1991.

[7] J. Whitman, The Ubiquity of the Gloss. Scripta, vol.2, pp95-121, 2011.

[8] M. Zisk, Research on Semantic Borrowing via Chinese Characters in Japanese. Dissertation. Tohoku University Graduate School of Arts and Letters. 2013. 